\title{
Hacia una Transformación de Espacios Relacionales: Estudio de Caso de una Experiencia en Educación en Derechos Humanos
}

\section{Towards a Transformation of Relational Spaces: A Case Study of an Experience in Human Rights Education}

\author{
Carlos Bustos $^{1, *}$, Silvia Castillo ${ }^{1}$, Simona Mayo $^{1}$, Jorge Soto ${ }^{2}$ \\ ${ }^{1}$ Universidad Católica Silva Henríquez, Chile \\ ${ }^{2}$ Universidad Diego Portales, Chile
}

\begin{abstract}
DESCRIPTORES:
Educación en derechos humanos

Educación continua Resignificación

Conciencia histórica

Cambio educativo

\section{RESUMEN:}

Durante los últimos años, se han llevado a cabo diversas acciones de promoción y educación en derechos humanos en Chile, las cuales buscan hacerse cargo de su insuficiente abordaje en la educación formal y en los procesos formativos en las instituciones públicas. Cuestión que se ve refrendada en las generalizadas violaciones a DDHH tras el estallido social de octubre 2019. En este marco, el presente trabajo se propone indagar en el impacto subjetivo de un Programa de Formación en Educación en Derechos Humanos de una universidad chilena orientado a profesionales de la educación. Desde un estudio de caso y un enfoque cualitativo, se produjo información a partir de grupos focales y producciones narrativas. A partir de un análisis de contenido, los resultados dan cuenta de las motivaciones, perfiles formativos y procesos de resignificación de los(as) participantes. Los resultados permiten problematizar respecto de las necesidades formativas y la emergencia de nuevos ángulos de construcción de conocimiento para programas EDH. En este sentido, se concluye que es clave incorporar estrategias pedagógicas que contribuyan a fortalecer estos programas y enfoque.
\end{abstract}

\section{KEYWORDS: \\ Human rights \\ education \\ Continuing education \\ Re-signification \\ Historical awareness \\ Educational change}

\begin{abstract}
:
During recent years, a number of different actions have been taken with respect to human rights promotion and education in Chile, which try to address the insufficient coverage in formal education, as well as formation processes in public institutions. This matter has come to light given the generalized human rights violations during the social outburst occurred in October 2019. Within this framework, the present study proposes an inquiry into the subjective impact of a formation program in human rights education for education professionals, carried out in a Chilean University. Applying a case study and qualitative approach, information was produced from group interviews and narrative productions. Based on the analysis of content, the results reveal the motivations, formative profiles and resignification processes of the participants. The results allow to problematize the formative needs and the emergence of new angles of knowledge construction for HRE programs. In this sense, it is fundamental to incorporate pedagogical strategies that contribute to strengthen these programs and the approach.
\end{abstract}

CÓMO CITAR:

Bustos, C., Castillo, S., Mayo, S. y Soto, J. (2021). Hacia una transformación de espacios relacionales: Estudio de caso de una experiencia en educación en derechos humanos. REICE. Revista Iberoamericana sobre Calidad, Eficacia y Cambio en Educación, 19(1), 5-21. https://doi.org/10.15366/reice2021.19.1.001

*Contacto: cbustosr@ucsh.cl

ISSN: 1696-4713

www.rinace.net/reice/

revistas.uam.es/reice
Recibido:

25 de febrero 2020

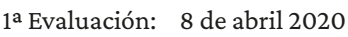

2a Evaluación: 29 de mayo 2020

Aceptado:

10 de junio 2020 


\section{Introducción}

Desde 1973, los Derechos Humanos (DDHH) se han instalado desde un relato histórico trascendental en la sociedad chilena a raíz de la violación sistemática de estos durante la dictadura militar y sus nefastas consecuencias políticas, sociales, culturales y psicológicas. Actualmente, la preocupación por estos derechos fundamentales se ha profundizado aún más a causa del reciente estallido social del 18 de octubre del 2019 y el actuar de las fuerzas del orden de Chile.

En este escenario histórico-concreto, de alta complejidad, la Educación en Derechos Humanos (EDH) como preocupación pedagógica ha venido desarrollándose como área de estudio de manera regular y progresiva a nivel internacional y nacional. Los últimos veinte años han sido prolíficos en investigación sobre el tema. Desde diversas disciplinas de las humanidades, ciencias sociales y la salud, entre otras, se ha comenzado a indagar en este enfoque que ha demostrado una singular capacidad para abordar distintas temáticas desde la mira de los derechos humanos. A modo de hitos recientes, en el año 2016 se realizó por primera vez en Latinoamérica la VII Conferencia Internacional de Educación en Derechos Humanos (IHREC), organizada en Chile por la Facultad de Derecho de La Universidad de Chile, a través de su Centro de Derechos Humanos y su Dirección de Extensión, en cooperación con la Western Sydney University. Previamente, este evento se había desarrollado en Australia, Sudáfrica, Polonia, Taiwán, Estados Unidos y los Países Bajos.

La actividad académica referida culminó con una publicación que aglutina numerosas experiencias de EDH (Villegas, Newfarmer y Francesconi, 2017) abordadas desde la literatura, el teatro, los sitios de memoria, la fotografía, el trabajo con el medio ambiente, los espacios carcelarios, incluso desde lo empresarial. Las investigaciones presentadas en este evento son un claro ejemplo de que actualmente la EDH es un ámbito de trabajo interdisciplinario y abordable desde múltiples perspectivas.

Desde el plano internacional, en Latinoamérica, la EDH se ha centrado principalmente en las dictaduras cívico-militares y en los conflictos armados, donde se ha desarrollado una línea de estudios en torno al concepto de Educación para la Paz (Álvarez-Maestre y Pérez-Fuentes, 2019; Paz y Díaz, 2019; Torres, 2019; Trimiño y Amézquita, 2018). En esta línea, por ejemplo, Martínez Sainz (2018) revisa, por medio de tres estudios cualitativos, los programas de EDH en relación con las violencias institucionales y el conocimiento profesional y las prácticas de los educadores en la implementación de estos programas en México. Candamil, Sánchez y Silva (2014) desarrollan una relevante revisión documental de los principales instrumentos de políticas públicas que determinan el diseño y formulación de estrategias educativas para EDH.

En relación con experiencias particulares, se relevan los estudios realizados en torno a la EDH vinculada a la historia reciente de Chile donde se ha abordado el periodo de la dictadura militar, en distintos niveles de la educación básica y media. Dentro de estos, la investigación de Santibáñez (2017), Toro (2017), Mercier, (2017) y Siemon (2017) son destacadas al momento de pensar en experiencias específicas desde la práctica educativa.

Con los procesos de redemocratización de la región, se desarrolla una serie de experiencias y trabajos sobre EDH a través de la creación de museos, archivos y sitios de memoria (Bascuñán, 2017; Morales y Sandoval, 2017; Silva e Ibáñez, 2015; Veneros y Toledo, 2009). Desde estos escenarios, emerge la necesidad de construir y fortalecer una política de educación de derechos humanos que cuente con experiencias relevantes como las de Argentina y Uruguay. Al respecto, el Informe sobre Educación en Derechos Humanos elaborado por el Instituto Interamericano de Derechos Humanos (IIDH) el año 2013, señala la necesidad de enfatizar en la enseñanza el conocimiento específico sobre derechos humanos en todos los niveles educativos en coherencia con metodologías apropiadas. De tal forma, esto se ha posicionado como un desafío para la región el consolidar la EDH como un saber pedagógico que impulse y aporte cambios curriculares y didácticos a través de políticas públicas educativas (IIDH, 2013).

Particularmente, Chile se ha destacado también por presentar estudios que han logrado mancomunar y poner en valor amplias propuestas y experiencias. Así, se relevan los aportes de Magendzo (2001, 2006, $2007,2010,2011$ ), quien en su trayectoria como investigador ha abordado la EDH desde lo curricular y desde la perspectiva de los docentes en la actualidad, en relación con las tensiones y desafíos que esta presenta al momento de diseñar propuestas educativas. Respecto de estas reflexiones, desde una pers- 
pectiva ética, Magendzo y Bermúdez (2017) han profundizado en proponer trabajar la EDH en articulación con una pedagogía crítica-dialógica.

Las percepciones de estudiantes y docentes no han estado ausentes en las recientes investigaciones en Latinoamérica. Ejemplo de ello es el trabajo de Carabantes (2014) en una universidad regional chilena donde revisa las reflexiones de estudiantes y egresados de pedagogía en relación a su formación en DDHH. Así también, Carabantes y Contreras (2017) estudian los sentidos que aportan las experiencias formativas en DDHH a la práctica pedagógica. Por su parte, la investigación de Cubillos-Vega (2020), desde un enfoque pedagógico crítico, aborda la incidencia de la EDH con estudiantes de Trabajo Social y analiza el aprendizaje y comprensión de las materias y las percepciones del estudiantado español.

Desde el área de la salud, se encuentra el novedoso trabajo de Valderrama (2020) que profundiza sobre los alcances y desafíos de la EDH en la formación de terapeutas ocupacionales por medio del abordaje de las percepciones de estudiantes. En un enfoque similar a nivel societal, Álvarez-Bravo (2019) da cuenta acerca de la construcción de sentidos que para la sociedad chilena tiene la educación y los derechos humanos, no solo a nivel sistémico, sino también en la cotidianidad.

La literatura entrega una robusta experiencia científica en torno a la EDH. La incorporación de conocimiento y comprensión de los derechos humanos, en los diferentes niveles educativos, se plantea como una necesidad imperiosa, puesto que constituye una oportunidad para concretar el ejercicio de estos (Naciones Unidas, 2012; Vega-Gutiérrez y Navaridas-Nalda, 2018).

En el caso particular de Chile, la distancia temporal entre la coyuntura de la dictadura militar y el actual escenario de vulneración de derechos humanos post estallido social, lo ubica en una situación de alta opacidad que lleva a la formulación de las siguientes preguntas, a propósito de los graves casos de violencia (Amnistía Internacional, 2019; Comisión Interamericana de Derechos Humanos [CIDH], 2019; Human Rights Watch, 2019; Instituto Nacional de Derechos Humanos [INDH], 2019; Naciones Unidas, 2019): ¿cuál fue el proceso de aprendizaje que vivió la sociedad chilena y sus diversos actores institucionales en relación con la violación sistemática de derechos humanos? ¿Cuál fue el impacto de las diversas comisiones, como la de Informe de la Comisión Nacional de Verdad y Reconciliación (1991) y el Informe de la Comisión Nacional sobre Prisión Política y Tortura (Valech I) (2004), en los procesos de aprendizaje de la sociedad? ¿De qué forma y a través de qué ángulos de construcción de conocimiento el sistema educativo en los gobiernos "democráticos" se ha hecho responsable de este campo de conocimiento y sus procesos de aprendizaje? ¿Qué papel ha jugado la educación superior y la educación continua en la formación en derechos humanos?

Todo este cúmulo de situaciones en las que se han visto silenciados los derechos de los pueblos fundamentan la necesidad de reforzar la EDH a nivel societal y de manera transversal e interdisciplinaria (INDH, 2012, 2013). No obstante, este significante requiere un horizonte de profundización de lo cotidiano e institucional, no como discurso separado del sujeto/a sino como práctica transformativa. Es aquí justamente donde yace el mayor desafío para la Educación Superior y la Educación Continua, ya que es aquí donde se forman los cuadros políticos y funcionarios del aparato estatal.

La concreción de una EDH exige así el uso de metodologías que vinculen la teoría y la práctica de los derechos humanos en contextos reales y concretos. La didáctica aparece como una dimensión relevante para llevar a cabo el proceso de enseñanza y de aprendizaje en el contexto de la EDH. Si bien los estudios revisados enfatizan en la incorporación de estrategias y recursos didácticos diversos, son escasas las investigaciones que analizan, particularmente, la práctica didáctica de este conocimiento pedagógico (sustentado en una perspectiva crítica). Por consiguiente, el presente artículo tiene como objetivo interpretar críticamente la implementación del Diplomado en EDH de una universidad chilena, desde la perspectiva de los(as) participantes, y comprender la (re)significación que le atribuyen a dicha experiencia formativa, desde la perspectiva de la conciencia histórica.

\subsection{La epistemología de la conciencia histórica y la didáctica del sentido como un enfo- que pedagógico para una EDH}

En el presente estudio, se toma como referente clave para la reflexión en torno a la EDH la epistemología de la conciencia histórica o presente potencial desarrollada por Hugo Zemelman. Esta sostiene que la realidad social está en constante movimiento y producto de ello se hace compleja su aprehensión en 
términos intelectuales, cognitivos, emocionales, axiológicos y volitivos. Zemelman (1992) advierte que si la realidad es pensada desde este dinamismo, cualquier delimitación conceptual o teórica que se haga de ella expresa un momento y también la posibilidad de otro nuevo.

Desde este enfoque, el conocimiento es concebido como una forma particular de apropiación que otorga al sujeto/a la capacidad de ubicarse en un momento histórico y la posibilidad de re-actuar sobre sus propias circunstancias (Zemelman, 1992). Esta noción tiene consecuencias en la ubicación del sujeto/a, respecto del conocimiento y la realidad, ya que le entrega un sentido en movimiento al acto de conocer y, simultáneamente, abre espacios de posibilidades de reconfiguración de la subjetividad.

Desde esta perspectiva, la conciencia histórica propone al sujeto construir conocimiento desde una exigencia propia de colocación frente a su contexto individual y colectivo dentro de la sociedad. A partir de este ejercicio, se concibe al sujeto/a desde sus límites y potencialidades y se propicia la habilidad de repensar lo establecido y renovarlo. En consecuencia, el sujeto/a no se acepta como agotado/a sino que adopta un razonamiento desde la necesidad de trascendencia y se exige a sí mismo transformar lo indeterminado en potencialidad (Zemelman, 2010). Según Zemelman (2011), se trata de recuperar al sujeto/a y al presente desde un anhelo de futuro, donde es capaz de recuperar su subjetividad y de valorizarse a sí mismo como persona que construye futuro.

En este marco, otra de las nociones que guían este estudio es la concepción de la didáctica del sentido (Quintar, 2009) surgida de reflexiones epistémicas y pedagógicas producto del cruce entre la epistemología de la conciencia histórica o presente potencial propuesta Zemelman y la pedagogía como ciencia crítica planteada por Juan Ruz (1992a, 1992b). La didáctica del sentido profundiza en una práctica educativa con significación histórica-social y capacidad de transformación. Al respecto, Quintar (2009) señala que existe en esta "la promoción del deseo del saber-de sí y del mundo, en sucesivos actos de con-ciencia histórico-social” (p. 40). De esta forma, asume la matriz epistémica de la conciencia histórica como un nuevo ángulo de construcción de conocimiento que permite articular la experiencia didáctica desde la realidad histórica contextual. Esta articulación otorga sentido y significado a los/as sujetos/as que la viven como experiencia y como sujetos/as históricos/as e historizados/as y les permite generar construcciones simbólicas que aportan a procesos de ruptura y resignificación a través de dispositivos didácticos.

Se observa en esta propuesta didáctica cómo se pone en tensión la subjetividad, las relaciones intersubjetivas, la realidad y las apropiaciones del conocimiento. Producto de esto, adquieren relevancia dos aspectos metodológicos que son dinamizadores de procesos de enseñanza transformativos: las preguntas reflexivo-problematizadoras y la didacto-biografía. Ambas conectan a los sujetos/sujetas desde su racionalidad, construida como fruto de sus historias de vida, y desde donde se develan sus representaciones simbólicas y acciones comunicativas.

\subsection{Derechos humanos y educación en derechos humanos}

Los derechos humanos constituyen el respeto a la dignidad como personas y todas las exigencias que de ellas deriven como, por ejemplo, la inclusión social, el ser parte y partícipe de una comunidad con igualdad de acceso a bienes disponibles materiales y simbólicos en un sentido amplio de las interacciones de estos elementos (Rodino, 2015). En consecuencia, la educación y la formación en derechos humanos son esenciales para la promoción del respeto universal y efectivo de todos los derechos humanos y las libertades fundamentales, de conformidad con los principios de universalidad, indivisibilidad e interdependencia de estos (Silva e Ibáñez, 2015).

En este marco, la EDH constituye un conjunto de actividades de formación, difusión e información cuyo objetivo es crear una cultura universal, a través de acciones y actividades que transmiten conocimiento y actitudes, cuya finalidad es: a) fortalecer el respeto de los derechos humanos y las libertades fundamentales; b) desarrollar plenamente la personalidad humana y el sentido de la dignidad del ser humano; c) promover la igualdad, la comprensión, la tolerancia, la igualdad de género y la amistad entre todas las naciones, las poblaciones indígenas y los grupos raciales, nacionales, étnicos, religiosos y lingüísticos; d) facilitar la participación eficaz de todas las personas en una sociedad libre; e) intensificar las actividades de Naciones Unidas en la esfera del mantenimiento de la paz (Naciones Unidas, 1996). 
Ahora bien, la EDH es relativamente reciente para el mundo y para América Latina y emerge como un mecanismo de defensa ante las violaciones sistemáticas de derechos humanos en la década de 1970 y 1980 (Azúa, 2011). Actualmente, una de las preocupaciones en este ámbito, lo constituye la estructura educativa y el currículum en tanto expresiones de la política pública, el efecto de estos dispositivos para moldear a los sujetos de derechos a través de organizaciones jerarquizadas, la ideología de la educación, los modelos de disciplina, las normas legales del Estado y sus regulaciones (Magendzo, 2012). Todas ellas tienen consecuencias en las epistemes institucionales y los ángulos de construcción de conocimiento de los sujetos y sujetas.

En la conformación de la EDH, uno de los principales hitos es la Conferencia Mundial de Derechos Humanos de Viena (1993), donde se señala que la promoción y protección de los derechos humanos es un asunto fundamental para la comunidad internacional, de forma tal que los Estados planteen una educación orientada al respeto de estos. Para ello, se propone la necesidad de su difusión con el objeto de entregar una información adecuada, tanto teórica como práctica, para la promoción y respeto de los derechos humanos de todas las personas, sin distinción alguna por motivos de raza, sexo, idioma o religión (Naciones Unidas, 1993). Una década después, se firmó la Declaración de Formación y Educación en Derechos Humanos que afirma que la educación en este ámbito es un derecho y debe ser garantizado por los Estados (Naciones Unidas, 2012). En consecuencia, en el año 2013 Naciones Unidas (2013) hizo un llamado a materializar procesos formativos en derechos humanos, llevar a cabo investigaciones, destacar y compartir buenas prácticas, incorporar y fortalecer metodologías pertinentes con la proyección de ser evaluadas, además de promover esta materia en los planes de estudio de los establecimientos y en los diferentes programas de formación. De esta forma, la EDH es relativamente reciente y, específicamente en Chile, el organismo que ha desarrollado acciones para su discusión y promoción a nivel institucional y estatal es el INDH, creado el año 2010.

\subsection{La formación continua en educación en derechos humanos}

La educación continua como campo de problematización para la actualización docente requiere profundizar en procesos de resignificación que permitan a los participantes de programas de educación continua no adquirir solo una acreditación, sino asistir a procesos que potencien los espacios relacionales educativos, en donde la conciencia histórica sea una orientación epistémica. Con ello, se espera que los y las profesionales puedan entablar una relación coherente entre su discurso y la acción en sentido transformativo y enriquecedor (Bustos y Castillo, 2017), sobre todo, en materia de derechos humanos, de acuerdo a los informes del INDH mencionados.

Actualmente, en Chile, existe una oferta amplia de diplomados sobre derechos humanos en ejecución; dentro de este campo, pueden mencionarse los programas de la Universidad de Chile, la Universidad Católica Silva Henríquez (en adelante UCSH), la Universidad Alberto Hurtado, la Universidad Academia de Humanismo Cristiano, la Universidad Católica de Temuco. Dichas instituciones se han planteado desde diferentes ejes temáticos como: la memoria y derechos humanos, ciudadanía y derechos humanos, derechos humanos para educadores en contextos interculturales, entre otros. A lo anterior, se agrega el programa ofrecido por el Ministerio de Educación titulado Derechos Humanos para docentes del sistema escolar impulsado a través del Centro de Investigación y Experimentaciones Pedagógicas (en adelante CPEIP) y el INDH.

Otras experiencias de EDH la constituyen los sitios de memoria que se construyeron donde antes hubo campos de concentración y exterminio y sitios de tránsito y de tortura durante la dictadura cívico-militar de Augusto Pinochet. Muchos de estos espacios fueron recuperados por organizaciones y comunidades de ex presos políticos, ejecutados y familiares de detenidos desaparecidos. Entre ellos, se encuentran el Parque por la Paz Villa Grimaldi, Londres 38, el Memorial de Paine y más de veinte sitios de memoria a lo largo del país que desarrollan actividades educativas en ámbitos no formales de gran relevancia en la construcción de una cultura de derechos humanos. 


\subsection{El diplomado de educación en derechos humanos de la Universidad Católica Silva Henríquez: Una experiencia desde los(as) sujetos(as) participantes}

La EDH para la UCSH constituye un imperativo ético para una institución que lleva el nombre de un defensor y promotor de los derechos humanos como lo fue el Cardenal Raúl Silva Henríquez. En este sentido, los derechos humanos son el eje principal de su trabajo educativo, como se afirma en sus Estatutos y Política de Investigación (UCSH, 2019).

En coherencia con esta preocupación, surge el Diplomado de Educación en Derechos Humanos en colaboración con el INDH, a partir de la necesidad de generar un programa de educación continua, cuyo propósito es aportar a la comprensión de la EDH como parte del derecho a la educación y, por consecuencia, como factor de calidad aplicable a aspectos de gestión, currículo, didáctica y de convivencia para la construcción también de una ciudadanía democrática y respetuosa de los derechos humanos (UCSH e INDH, 2018). De esta manera, el programa está destinado a profesionales que se desempeñan en todos los niveles del sistema educativo.

El Programa se inició el año 2016 y hasta la fecha tiene cuatro versiones concluidas (abril 2020). La modalidad es semipresencial y posee 35 cupos abiertos a profesionales de la educación del país. El plan de estudios cuenta con un total de 250 horas, con trabajo presencial y en línea. Está estructurado según el perfil de competencias de egreso en tres módulos: a) Introducción a los DDHH y Derecho a la Educación, b) Estrategias de Educación en Derechos Humanos y c) Los Derechos Humanos y las estrategias de gestión, currículum, didáctica y convivencia para las comunidades educativas.

Esta propuesta cuenta con criterios definidos y un proceso de selección de participantes que considera un enfoque de derechos humanos. En sus cuatro versiones, ha presentado buenos resultados en términos de convocatoria (29 participantes como promedio) — con asistencia permanente de profesionales de diferentes regiones-, siendo alta las tasas de retención y titulación, así como también de evaluación por parte de los mismos, como se da cuenta en los informes elaborados anualmente (Bustos, 2018)

\section{Método}

El diseño de la investigación corresponde a un estudio de caso intrínseco (Stake, 2007). El caso en cuestión lo constituye el Diplomado de Educación en Derechos Humanos de la UCSH, en su tercera versión (2018). Como señala Kazez (2009), el estudio de caso es una aproximación intensiva y contextual, sea con fines de descripción de procesos sociales y/o teorización sobre los mismos. En particular, se trata aquí de la interpretación crítica de la experiencia formativa sobre EDH, desde la perspectiva de los participantes.

El enfoque metodológico es de tipo cualitativo con un alcance descriptivo e interpretativo, con énfasis en los procesos de escucha y diálogo como base para producción y validación de conocimiento. La producción de información tiene un enfoque retrospectivo, en cuanto se orienta a la reconstrucción de los ejes desarrollados por el Diplomado y la significación de la experiencia formativa en EDH, por parte de los/ as participantes. La producción de información considera la triangulación de fuentes documentales y la aplicación de dispositivos grupales.

El material documental refiere a las cartas de motivación de 35 postulantes de la tercera versión, así como de la ruta de aprendizaje, la cual corresponde a un dispositivo narrativo elaborado al cierre del proceso, en donde estos autoevalúan y significan su experiencia formativa en EDH. Por otra parte, se implementa un grupo focal con los/as estudiantes (n10) que finalizaron el programa en su tercera versión. Respecto de su composición, se procuró representar la heterogeneidad del grupo, a partir su variabilidad territorial, ocupacional y de género.

El análisis, tanto de las fuentes documentales como de las transcripciones del grupo focal, corresponde a un análisis cualitativo de contenido mediante codificación y síntesis temática, con una lógica combinada (deductiva-inductiva) y asistido por el programa Atlas ti. 7.2. Las dimensiones de análisis consideran aspectos curriculares; pedagógicos y didácticos; organizativos; contextuales; los significados (re)construidos en torno a la EDH y sus vínculos con las estrategias formativas. En los resultados que a continuación se presentan, focalizamos en estas últimas dos dimensiones. 


\section{Resultados}

En este apartado, se presentan las perspectivas (retrospectivas) de los(as) egresados(as) de la tercera versión del Diplomado por medio de una síntesis interpretativa respecto de: i) las motivaciones y expectativas previas al ingreso del programa, ii) los procesos transversales de (re)significación de la experiencia formativa y iii) la descripción de dos perfiles discursivos en los estudiantes sobre la experiencia.

\subsection{Motivaciones y expectativas iniciales}

Las motivaciones y expectativas se tomaron de una carta que forma parte del proceso de postulación al Diplomado de la tercera versión (2018). Se realizó una síntesis temática de las mismas, según la relación entre: a) las motivaciones vinculadas al contexto de referencia en que se inscribe una necesidad formativa en EDH y, b) las expectativas respecto de la formación y su foco de proyección.

\subsubsection{El contexto de relevancia laboral y de expectativas de impacto según foco profesional-nor- mativo ofoco ético-político}

Refiere a motivaciones localizadas o circunscritas en el contexto laboral inmediato de las y los participantes, vinculadas a la necesidad de obtener herramientas para la incorporación del enfoque en DDHH. Asimismo, se plantea como una necesidad de desarrollo profesional y personal frente a la ausencia de herramientas en la formación universitaria previa, y una exigencia de articular adecuadamente las bases normativas en derechos humanos en los contextos educativos. Las siguientes citas dan cuenta de lo señalado:

Por tal razón, una vez al año generamos cambios y actualizaciones del manual de convivencia, mejoramos los proyectos de integración pensando en la interculturalidad y en especial el trabajo de sensibilización dentro de los colegios a la tolerancia y respeto. Pero esta tarea se vuelve difícil al no contar con las herramientas metodológicas o teóricas relacionadas con los derechos universales e infantiles. (Participante 3, comunicación personal)

Hoy en día considero una necesidad este tipo de formación académica, primero, porque mi pregrado no fui formada bajo esta mirada y siento la necesidad de hacerlo; $y$, segundo, porque el entregar un enfoque de derechos dentro de la educación está siendo cuestionada debido a la ausencia de herramientas de cómo entender a la normativa vigente nacional, así como la internacional en esta temática. (Participante 11, comunicación personal)

Se expresa con claridad la necesidad formativa y normativa para fortalecer los espacios laborales en torno a un enfoque de $\mathrm{EDH}$, que ha estado ausente en la formación profesional. Por otra parte, se distinguen motivaciones situadas en este contexto laboral que, sin embargo, ponen énfasis en expectativas que aspiran a la transformación de personas y de alcance comunitario en dichos contextos, como se señala a continuación:

Mi principal motivación al iniciar este diplomado, es continuar adquiriendo herramientas prácticas que promuevan el crecimiento y la dignidad tanto de los usuarios como de las comunidades donde trabajo. (Participante 18, comunicación personal)

\subsubsection{Motivaciones situadas en el contexto societal, según énfasis en aspectos ético-políticos}

Para el ingreso al Diplomado, se requieren ciertas declaraciones en las motivaciones de los(as) participantes que justifiquen su incorporación; significantes como "actualidad" o "realidad actual" son mencionados y caracterizados como el resultado de vulneraciones históricas de derechos. En consecuencia, estas se abordan desde una perspectiva de EDH, pues se vinculan a la necesidad propuesta de un "mejor lugar para vivir", para "la justicia social” y una "sociedad democrática", entre otros tópicos. Se hace referencia a motivaciones vinculadas a un diagnóstico social más amplio y a un cambio paradigmático en la educación para enfrentarlo, tal como se expresa en la siguiente cita:

Como participante activa en el mundo educativo de nuestro país, creo que, si bien resulta un poco utópico pensar que la escuela por sí sola pueda cambiar el mundo, sin duda, es imposible pensar en un cambio sin ella. Siendo así, considero que, en este momento, es necesaria la 
instauración de un nuevo paradigma educativo, cuyos fundamentos apuesten por la construcción de un mejor lugar para vivir. (Participante 5, comunicación personal)

Las motivaciones y expectativas que declaran constituyen un requisito de entrada y articula con los nuevos ángulos de construcción de conocimiento que aporta el diplomado. Esto con el objetivo de elaborar un diagnóstico participativo que derive en un proyecto de intervención en EDH para aportar al enriquecimiento, en el corto plazo, del escenario de trabajo de los participantes.

\section{2. (Re) significación de la experiencia, aspectos transversales: Hacia una cultura de transformación en EDH}

Respecto de la significación que las y los estudiantes otorgan a la experiencia formativa del diplomado, es decir, la forma en cómo reconocen afectaciones relevantes en el plano profesional y personal, podemos destacar aspectos transversalmente reconocidos como significativos, por el grupo, en su formación y a los que se vinculan dispositivos de resignificación desde la epistemología de la conciencia histórica.

\subsubsection{Hacia una cultura de transformación en educación en derechos humanos}

De manera conjunta, las y los estudiantes reconocen un cambio en su perspectiva previa sobre los DDHH y su relación con la educación, aun en quienes manifiestan poseer algún bagaje previo. Refieren así aprendizajes que movilizan nuevas comprensiones, tensionando a veces sus conocimientos previos. Evidencian este movimiento en su ruta de aprendizaje a partir de expresiones del tipo: darme cuenta, cambiar el swicht, resignificar, vivir un aprendizaje, comencé a visualizar otras aristas, entre otras.

Una forma de sintetizar lo anterior, puede describirse en términos del movimiento de los(as) sujetos(as): transición o desplazamiento desde una concepción circunscrita a temáticas o ámbitos específicos (su dimensión legal por ejemplo) a una comprensión de carácter más integral de los DDHH y su relación con la educación. Este lugar de llegada, en sus palabras, se puede señalar como la comprensión desde una cultura de educación en Derechos Humanos. Esta noción consideraría una diversidad de actores, situaciones y escalas en que se manifiestan los DDHH y con repercusiones para la práctica profesional de cada participante, como se afirma en la siguiente cita:

Entré al diplomado con una expectativa más bien legalista, es decir, conocer los distintos aspectos legales de DDHH, cómo funcionan y se asimilan, así como también una visión más de identificación de vulneración. Dicho de otra forma, bastante cerebral la aproximación a los DDHH (...) En resumen, ha sido una buena ruta, que ha modificado mi idea_de la temática, relevando la importancia de que la EDH está permanentemente en la vida cotidiana y nos presenta un gran desafío porque implica activar en míy en el otro lo racional y lo emocional, vivencial. (Participante 23, ruta de aprendizaje)

En esta perspectiva, se destacan dos aspectos relacionados con: a) una nueva lectura de los contextos cotidianos y estructurales (y sus articulaciones) y, b) el reconocimiento y afirmación de la propia capacidad de agencia para la promoción y EDH, así como de transformación desde y en los propios mundos de vida (la vivencia). Las citas que a continuación se presentan evidencian ambos aspectos: "Durante las clases, pude resignificar varios conceptos ideas, que no sabía que los tenía, respecto a la cotidianidad, a lo violento, discriminador y desigual que parece este país y sociedad" (Participante 17, comunicación personal). En la misma línea, dentro de una conversación colectiva se señala:

A mí lo que me sorprendió es la reflexión permanente te lleva a empezar a mirar desde otra perspectiva, te sitúa en el análisis de la situación de la vida desde otro espacio y creo que esa es un poco la convergencia de todos los elementos que constituyeron el diplomado. Entonces frente a cualquier situación o que uno mira, no sési les pasa a ustedes, está permanentemente el tema o en relación a la infancia o el estar mirando con otros ojos, porque se incorporan fuertemente esos elementos. Creo que se genera un proceso de transformación importante. (Participante 8, Grupo focal, comunicación personal)

Se advierte así la tensión que está presente entre el discurso y los sujetos(as), por tanto, la coherencia y los imperativos éticos son fundamentales para profundizar los procesos, no solo en el macro espacio sino 
justamente en la cotidianidad. De esta forma, va tomando cuerpo el concepto de conciencia histórica en el que está implícito el doble movimiento del sujeto/a.

Este agenciamiento percibido se apoya en el reconocimiento de las herramientas adquiridas en el Diplomado, particularmente, en los espacios de práctica profesional, tales como la comprensión de los procedimientos de identificación y abordaje de vulneraciones, tal como se expresa en esta cita:

Lo otro es el énfasis para poder concretar lo que son la identificación de vulneración de derechos, el cómo concretar, por ejemplo, poder entender el derecho humano en la realidad actual. Creo que eso también es valioso, que no se quedó solamente en lo macro, sino que fue a lo micro, en nosotros también poder tener herramientas para poder identificar dónde hay una vulneración de derechos, cuáles son los procedimientos, el por quéy el para qué. Creo que eso igual es importante, porque en el fondo nos quedamos con herramientas para poder bajarlo a nuestras propias realidades. (Grupo focal 3, comunicación personal)

\subsection{Factores de la propuesta formativa}

Parece relevante, para tener una comprensión de los procesos de resignificación de los(as) participantes respecto de la $\mathrm{EDH}$, las valoraciones que hacen de algunas dimensiones de la propuesta formativa. A continuación, se desglosan las más relevantes:

\subsubsection{Pedagogía de la vivencia: Lo sentir pensante y la escucha}

Existe una alta valoración del enfoque pedagógico del Diplomado, producto de la permanente integración de elementos conceptuales y vivenciales que permitió afirmar una apreciación generalizada entre las y los participantes de aprendizajes significativos. Dentro de ello, también destacan características de un intencionado clima emocional, de apertura y de escucha, el que a la vez que tensionaba, invitaba a la reflexión desde un sentir pensante. Esta intersubjetividad en instancias de retroalimentación (de docentes y entre pares) permitió lograr altos grados de resonancia didáctica a nivel personal y en la construcción de significados, como se puede apreciar en la siguiente reflexión:
Ahora bien, el trabajo del módulo 2 y 3, ha sido más complejo porque me han sorprendido las emociones. Me costó cambiar el switch y abrir otra conciencia de percepción de los DDHH, que no es solo conocerlo, sino que su importancia es sentir, un sentir pensante. (Participante 23, comunicación personal)

\subsubsection{Contribuciones de una didáctica implicativa y transformadora}

Dentro de este enfoque pedagógico, se destaca la relevancia de los dispositivos didácticos por su capacidad para implicar al sujeto y su historia en la experiencia educativa presente. Se refieren así las y los participantes, directamente, a una didáctica innovadora y transformadora, tanto en relación con sí mismos como con su potencial de transformación y transferencia en los contextos profesionales y futuros en EDH. Las siguientes citas dan cuenta de lo señalado:

Es decir, existió uso de variados métodos que invitaron a pensar sobre nosotros mismos y nuestra realidad social. Esta conjunción de conocimiento (interno y externo) otorga pertinencia y significancia a los aprendizajes, ya que logra situar la teoría en la práctica. (Participante 20, comunicación personal)

A mí me dejan todas las experiencias didácticas, que no son las típicas las que más me removieron, y las estoy tratando de llevar a cabo en mi práctica diaria profesional y personal. Lo que hace S. lo que hace C. y la P. hacen desmarcarse de las clases expositivas, en los casos necesarios, y cuando son intervenciones de actividades que salen fuera de lo común, es lo que me deja a mi para aprender, lo que me mueve y es lo que debería mantenerse. (Participante 6 , Grupo focal, comunicación personal)

La expresión de los(as) participantes hacen implícitamente referencia al doble movimiento del sujeto(a) en su subjetividad por medio de la tensión de sus procesos de configuración o reconfiguración desde el pensarse a sí mismo. También, destaca la relación que tiene con el otro desde sus diálogos y expresiones 
verbales, no verbales y paraverbales. Todo esto expresado desde la conciencia histórica y con dispositivos que vienen desde la didáctica del sentido.

\subsection{Dos perfiles discursivos: Pragmáticos y problematizadores}

Si bien los aspectos antes descritos son transversalmente significativos para el conjunto de estudiantes, en su discurso grupal se pueden identificar al menos dos perspectivas que se diferencian, tanto por los aspectos que más valoran del Diplomado y su contribución, como por su adecuación al discurso implícito del programa formativo. De esta forma, se configura un perfil que se define como el de los pragmáticos frente a otro que puede denominarse los problematizadores. Estos se describen sucintamente a continuación.

\subsubsection{Pragmáticos}

Se caracterizan por valorar los aprendizajes en relación con su aplicabilidad directa y concreta en situaciones de la práctica profesional, por tanto, están más centrados en los contenidos explícitamente relacionados con el marco de los derechos humanos, los criterios y procedimientos para la identificación de su vulneración y formas de actuación. A continuación, es posible observar algunas de estas percepciones de los estudiantes:

En el módulo uno la verdad es que no tengo mayores reparaciones, porque fue bastante concreto y yo creo que a varios nos ayudó tanto las clases, los ejercicios que hicimos, los ejercicios formativos que hicimos y el trabajo final a identificar inmediatamente una vulneración y cuál era el procedimiento. (Participante 8, Grupo focal, comunicación personal)

Dentro de esta perspectiva, se plantea una necesidad, por una parte de: a) una mayor explicitación de la articulación y una secuencia anticipadamente más lineal del plan formativo y, b) una mayor comodidad con los temas y conceptos acotados y circunscritos, frente a la incorporación de ámbitos y problemáticas que, a su juicio, abren aspectos más complejos de abordar en el marco de un Diplomado en EDH. Siempre, desde la consideración de la diversidad del estudiantado, y la suya propia, como una condicionante, tal como lo expresa la siguiente cita:

Sí, de hecho, para mí era muy importante que me presentaran un orden previo, esto no significa que no estuviera contenta; postulamos muchas personas que querían estar, entonces también es muy complejo lograr ahondar criterios con una diversidad de personas y de fuentes laborales distintas, entonces quería como aclarar eso. Mi satisfacción al haber pasado por este programa es ciento por ciento satisfactorio, si bien hay como detalles que en el fondo para eso estamos aqui hoy día es como para aportar. Lo que transité en el diplomado me sirve en mi trabajo, yo lo reflexiono y lo llevó todos los días. (Participante 7, comunicación personal)

\subsubsection{Problematizadores}

Por otra parte, hemos descrito como un perfil problematizador, a un discurso de estudiantes en el que existe una alta valoración de aprendizajes surgidos del encuentro con problemáticas y marcos de referencia que desbordan lo que, en un principio, esperaban en el marco estricto de un Diplomado en EDH. Encuentro que identifican con el desarrollo de un pensamiento crítico o un desplazamiento paradigmático, como se expresa en la siguiente cita:

A mí me pasó que empecé a visualizar otras aristas que empezaron a ir más allá de lo que yo tenía en un principio, que era esto de educación en derechos humanos, y después empezó a aparecer este tema de la interculturalidad que yo creo es un tema bastante más amplio y que le da también otro marco teórico a lo que estábamos hablando, y finalmente me encontré en una suerte de pensamiento crítico,que ya finalmente me di cuenta que también ya estaba en otro paradigma... la excusa era lo de los derechos humanos para terminar en un pensamiento crítico. Fue una buena excusa partir hablando de derechos humanos y terminar mirándolo desde una perspectiva crítica, entonces a mí sí me hace mucho sentido lo que se hizo, porque si fuera solo sobre derechos humanos, la verdad, que yo creo que con el módulo uno se acaba 
el diplomado, porque no hay más que hacer. (Participante 5, Grupo focal, comunicación personal)

En este perfil, cierto carácter inductivo o no lineal de la experiencia formativa no se tematiza en tanto problema, al contrario, se entiende como una apertura de posibilidades y nuevas comprensiones. Se valora la apertura a temáticas y nuevos marcos conceptuales subyacentes, en tanto expresión de una mirada compleja que desborda y enriquece una visión sobre los DDHH y la EDH. Esto es asumido positivamente como una interpelación y un desafío para una mayor profundización y elaboración.

Estos dos perfiles contrapuestos representan dos perspectivas entre las que se encuentran matices, intersecciones y consensos relativos a los aspectos transversalmente valorados del Diplomado. No obstante, resulta interesante que estos dos perfiles tengan una muy buena valoración del diplomado en su integralidad y sientan de entera pertinencia su participación en él. Se puede suponer, por lo antes señalado, que esta adhesión se relaciona con su experiencia significativa con la propuesta educativa del programa.

\section{Discusiones y conclusiones}

Los hallazgos de este estudio permiten comprender la relevancia de incorporar el conocimiento y la comprensión de los derechos humanos en el ámbito educativo, en sus distintos niveles, dado que constituye una posibilidad de poner en práctica el ejercicio de estos, tal como lo señala la Declaración de EDH y diversos estudios (Gauché, 2012; Nin y Lorda, 2019; Osorio, 2014; Vega-Gutiérrez y Navaridas-Nalda, 2018). Dicha necesidad es parte de las motivaciones de los y las participantes del Diplomado, quienes advierten la oportunidad de fortalecer su desarrollo profesional y personal a propósito de su ausente formación universitaria en esta materia. Estos resultados son coherentes con los Informes del INDH $(2011,2014)$, cuyos datos revelan que la discusión e implementación de una EDH, con todas sus limitaciones y críticas, se ha focalizado en el sistema escolar principalmente.

Las motivaciones de entrada revisadas en este estudio llevan a equilibrar dos supuestos: i) puede ser un riesgo la burocratización de los derechos humanos en las instituciones educativas formales y no formales, o bien, derivar en una juridización no reflexiva de las prácticas profesionales (normativa-punitiva); y ii) puede conducir a un discurso de los derechos humanos muy amplio, voluntarista y sin una aplicación concreta en la instituciones y su cotidianidad.

Un aporte concreto de la presente investigación a los puntos referidos se da en el ámbito de la didáctica diseñada desde la epistemología de la conciencia histórica. El tratamiento de los contenidos, los dispositivos didácticos, los recursos educativos y la evaluación constituyen una contribución a la docencia. Esto se expresa en el discurso de los y las participantes, sobre todo, en los procesos de resignificación que experimentan. De acuerdo con el INDH (2018), la EDH exige metodologías que articulen la teoría y la práctica de los derechos humanos en contextos reales y prácticas de la vida cotidiana; sin embargo, dicho informe revela la falta de formación en contenidos y metodologías para la formación en DDHH en la Educación Superior.

Las novedades tras el análisis ponen en relieve la importancia de abordar la EDH desde una perspectiva crítica, que interpele la subjetividad en relación con el otro(a). De ahí, entonces, la necesidad de que quienes forman en EDH incorporen didácticas críticas que, junto con la identificación de la vulneración de derechos, provoquen rupturas epistémicas orientadas a cuestionar las relaciones de poder, las discriminaciones, las formas de colonialismo, las desigualdades, la segregación y las prácticas exclusoras, tal como ocurrió en el Diplomado.

Al respecto, los hallazgos dan cuenta de que los y las participantes vivencian procesos de resignificación en torno a su papel como sujetos/as en despliegue de posibilidades para contribuir a la reconstrucción de espacios relacionales micro, meso y macrosociales. Advierten la tensión que está presente entre el discurso y el/la propio(a) sujeto(a), por tanto, la coherencia y los imperativos éticos son fundamentales para problematizar la manera en que se habita la cotidianidad. De esta forma, va tomando cuerpo el concepto de conciencia histórica en el que está implícito el doble movimiento del sujeto(a) (Zemelman, 2011): por un lado, pone en tensión sus procesos de configuración o reconfiguración desde el pensarse a sí mismo; y, por otro, destaca la relación que establece/construye con el otro/a desde sus diálogos y expresiones ver- 
bales, no verbales y paraverbales. A partir de una pedagogía de la potenciación y una didáctica del sentido (Quintar, 2009) se fortalece la autonomía para construir proyectos de intervención de futuro mediante diversos dispositivos.

Así, fortalecer las metodologías y recursos de enseñanza resulta clave para materializar una EDH. Aunque no existen fórmulas para enseñar los contenidos de los DD.HH. y cómo ejercerlos y promoverlos, la experiencia acumulada plantea la necesidad de incluir estrategias que promuevan la participación, generen condiciones para que puedan ejercerse tales derechos (INDH, 2014, 2018) y fomenten la colaboración en función de una autonomía reflexiva en equipos a fin de realizar una lectura de la realidad que derive en posibilidades y escenarios de transformación (Figueroa, Soto y Sciolla, 2016). Ello implica un cambio de perspectiva, como lo expresan los y las participantes del Diplomado.

Entre sus motivaciones, destaca la potencialidad de una EDH para contribuir a la transformación social, en concordancia con Magendzo (2009) y Rodino (2015). Este aporte encuentra asidero si tiene un alcance comunitario, como es relevado. Esto demanda una coherencia entre el discurso y la práctica, de tal modo que la comprensión de los DD.HH. se materialice en las prácticas cotidianas. La construcción del proyecto de intervención y la evaluación final del Diplomado se orientaron a este objetivo, aportando a la dimensión ético-política de una EDH.

En este sentido ético-político, una de las preocupaciones del programa es el componente didáctico por ser un escenario donde hay configuraciones del discurso pedagógico que articulan concepciones teóricas, representaciones e imaginarios (Bustos y Castillo, 2017), considerando a su vez, que una EDH de carácter implicativa requiere de una bidireccionalidad, procesos reflexivos y metodologías activas participativas (De Oña y García, 2016).

Los resultados revelan una alta valoración del enfoque pedagógico dada la articulación entre lo conceptual y lo vivencial vinculado a la indagación de los y las participantes en sus subjetividades y diálogos intersubjetivos; el clima emocional de apertura a la escucha como dispositivo didáctico permitió una conexión con su historia y memoria y la posibilidad de "compartir y compartirse".

A manera de cierre las evidencias nos indican que en la experiencia política y relacional de la sociedad chilena en torno a los derechos humanos, se manifiesta una contradicción entre el discurso público de la institucionalidad del Estado y la realidad, expresada en las diversas violaciones a los derechos humanos luego del retorno a la democracia, principalmente, hacia el pueblo Mapuche y dirigentes sociales. Dicha situación se intensifica luego del estallido social del 18 de octubre, pasando a ser sistemática, de acuerdo a variados informes de organismos internacionales y del INDH. Además, evidencia que las políticas de EDH de los diversos gobiernos no han provocado un cambio cultural al interior de la institucionalidad del Estado y las fuerzas de orden, muy por el contrario, han sido frágiles y se han mantenido esencialmente en lo discursivo. En consecuencia, se requieren programas de EDH que reduzcan la brecha entre el discurso y la realidad.

A partir de los resultados de esta investigación y como proyección de ella, emerge la necesidad de indagar en el impacto del Diplomado a través de acciones de sus egresados y egresadas (117) y sus proyectos de intervención institucional. Dicha proyección respondería a las limitaciones de este estudio, que no aporta información sobre las repercusiones de la resignificación en los contextos educativos donde habitan los y las participantes del Diplomado. Un análisis de ello permitirá seguir profundizando y articulando una $\mathrm{EDH}$, que aporte al fortalecimiento del discurso de las políticas públicas y acciones del Estado para el resguardo de la sociedad.

\section{Agradecimientos}

Agradecemos al Instituto Nacional de Derechos Humanos por el trabajo conjunto en la realización del Diplomado en Educación en Derechos Humanos y por las becas que otorgó para facilitar el acceso a estudiantes interesado/as de todo el país. A la Dirección de Vinculación con el Medio y al área de Educación Continua de la Universidad Católica Silva Henríquez por su colaboración en el desarrollo y gestión del diplomado. Y finalmente, a los y las profesionales de la educación de las cuatro versiones de este programa que contribuyeron con sus testimonios y reflexiones para la realización de este primer estudio. 


\section{Referencias}

Álvarez-Bravo, P. (2019). Educación y derechos humanos en Chile, una relación necesaria. Revista Educación, 43(1), 592-604.

https://doi.org/10.15517/revedu.v43i1.29966

Álvarez-Maestre, A. J. y Pérez-Fuentes, C. A. (2019). Educación para la paz: Aproximación teórica desde los imaginarios de paz. Educación y Educadores, 22(2), 277-296.

https://doi.org/10.5294/edu.2019.22.2.6

Amnistía Internacional. (21 de noviembre de 2019). Chile: Política deliberada para dañar a manifestantes apunta a responsabilidad de mando. Amnistía Internacional.

https://amnistia.cl/noticia/chile-politica-deliberada-para-danar-a-manifestantes-apunta-a-responsabilidad-de-mando/

Azúa, E. (2011, octubre). La educación en derechos humanos. Ponencia presentada en el Seminario Internacional Universidad y Derechos Humanos. Santiago de Chile. https://bibliotecadigital.indh.cl/handle/123456789/43

Bascuñán, K. (2017, diciembre). La fuerza de un sitio de memoria. Trayectoria y desafíos de la propuesta educativa del parque por la Paz Villa Grimaldia. Comunicación presentada en la VII Conferencia Internacional de Educación en Derechos Humanos (IHREC). Santiago de Chile.

https:/www.uchile.cl/publicaciones/139918/vii-conferencia-internacional-de-educacion-en-derechos-humanos-ihrec

Bustos, C. (2018). Informe $N^{\circ} 1,2$ y 3 del diplomado de educación en derechos humanos al INDH. Universidad Católica Silva Henríquez.

Bustos, C. y Castillo, S. (2017). Interculturalidad, sujeto y pedagógica para una universidad abierta al futuro. En C. Valdés (Comp.), Posibilidades y utopías hacia una universidad intercultural (pp. 119-134). Ediciones UCSH.

Candamil Pinzón, J. G., Sánchez Avella, C. A. y Silva Medina, Y. L. (2014). Educación en derechos humanos: Revisión en perspectiva desde Europa, Asia Central, Norteamérica, Latinoamérica y Colombia. Revista Vía Iuris, 16, 87-117.

Carabantes, E. (2014). Reflexiones de estudiantes y egresados de pedagogía respecto a su formación en derechos humanos. Temas de Educación, 20(2), 125-141.

Carabantes, E. y Contreras, S. (2017). Núcleos de sentido que emergen desde la experiencia de formación en derechos humanos en docentes en ejercicio y estudiantes de pedagogía de una universidad estatal de Chile. Revista de Estudios Pedagógicos, 43(3), 83-101. https://doi.org/10.4067/S0718-07052017000300005

Cubillos-Vega, C. (2020). Educación en derechos humanos con enfoque pedagógico crítico: Estudio de caso. Trabajo Social, 22(1), 177-200.

https://doi.org/10.15446/ts.v22n1.77786

Comisión Interamericana de Derechos Humanos. (6 de diciembre de 2019). CIDH condena el uso excesivo de la fuerza en el contexto de las protestas sociales en Chile, expresa su grave preocupación por el elevado número de denuncias y rechaza toda forma de violencia. $O E A$.

http://www.oas.org/es/cidh/prensa/comunicados/2019/317.asp

Comisión de Verdad y Reconciliación. (1991). Informe de la comisión nacional de verdad y reconciliación. Corporación Nacional de Reparación y Reconciliación.

http://www.derechoshumanos.net/lesahumanidad/informes/informe-rettig.htm

Comisión Nacional sobre Prisión Política y Tortura. (2004). Informe de la comisión nacional sobre prisión política y tortura (Valech I). Ministerio del Interior - Comisión Nacional sobre Prisión Política y Tortura. https://bibliotecadigital.indh.cl/handle/123456789/455

CNN Chile. (13 de noviembre de 2019). Confirman veracidad de audio en el que general Rozas asegura que no dará de baja a ningún carabinero. CNN Chile.

https:/www.cnnchile.com/pais/audio-general-rozas-respaldo-carabineros_20191113/ 
De Oña, J. M. y García, E. (2016). Proyecto escuela: Espacio de paz. Reflexiones sobre una experiencia en un centro educativo. REICE. Revista Iberoamericana sobre Calidad, Eficacia y Cambios en Educación, 14(2), 115-131. https://doi.org/10.15366/reice2016.14.2.007

Figueroa, I., Soto, J. y Sciolla, B. (2016). Dinámicas de recepción, apropiación y contextualización del enfoque de la guía para la inclusión educativa en escuelas municipales de una comuna de la región metropolitana. Pensamiento Educativo. Revista de Investigación Educacional Latinoamericana, 53(1), 1-13. https://doi.org/10.7764/PEL.53.1.2016.16

Gauché, X. (2012). Pensando en los cambios a la educación en Chile desde el paradigma internacional de la educación en derechos humanos. Revista de Derecho Público, 77, 321-353.

Human Rights Watch. (26 de noviembre de 2019). Chile: Llamado urgente a una reforma policial tras las protestas. Human Rights Watch.

https://www.hrw.org/es/news/2019/11/26/chile-1lamado-urgente-una-reforma-policial-tras-las-protestas

Instituto Nacional de Derechos Humanos. (2011). Diagnóstico de la inclusión de la educación de derechos humanos en los textos escolares vigentes en el año 2011. Instituto Nacional de Derechos Humanos.

https://bibliotecadigital.indh.cl/handle/123456789/98

Instituto Nacional de Derechos Humanos. (2012). Informe anual 2012. Situación de los derechos humanos en Chile. Instituto Nacional de Derechos Humanos. https://bibliotecadigital.indh.cl/handle/123456789/296

Instituto Interamericano de Derechos Humanos. (2013). El derecho a la educación en derechos humanos en la educación en las Américas 2000-2013.

https://www.iidh.ed.cr/IIDH/media/1517/informe-interamericano-el-derecho-a-la-edh-2013.pdf

Instituto Nacional de Derechos Humanos. (2014). La educación en derechos humanos en universidad: Revisión crítica de cursos para la elaboración de un programa de formación básica. Instituto Nacional de Derechos Humanos. https:// bibliotecadigital.indh.cl/handle/123456789/954

Instituto Nacional de Derechos Humanos. (2018). Informes de encuentros de formación de formadores en derechos humanos. Instituto Nacional de Derechos Humanos. https://bibliotecadigital.indh.cl/handle/123456789/1143

Instituto Nacional de Derechos Humanos. (2019). Informe anual situación de los derechos humanos en Chile 2019. Instituto Nacional de Derechos Humanos.

https://bibliotecadigital.indh.cl/bitstream/handle/123456789/1701/Informe\%20Final-2019.pdf?sequence=1\&isAllowed=y

Kazez, R. (2009). Los estudios de casos y el problema de la selección de la muestra: Aportes del sistema de matrices de datos. Subjetividad y Procesos Cognitivos, 13(1), 71-89.

Magendzo, A. (2001). El derecho a la educación: Una reflexión desde el paradigma crítico y la educación en derechos humanos. Educación: Una Cuestión de Derecho, 2, 73-89.

Magendzo, A. (2006). Educación en derechos humanos: Un desafío para los docentes de hoy. Editorial LOM.

Magendzo, A. (2007). La educación en derechos humanos: Diseño problematizador. DEHUIDELA, 15, 65-74.

Magendzo, A. (Ed.). (2009). Pensamiento e ideas fuerza en la educación en derechos humanos en Iberoamérica. Editorial SM.

Magendzo, A. (2010). Dilemas y tensiones curriculares y pedagógicas de la educación en derechos humanos. Revista IIDH, 52, 321-328.

Magendzo, A. (2011). Educación en derechos humanos: Un desafío impostergable. En F. Cousiño Donoso y A. M. Foxley Rioseco (Eds.), Politicas públicas para la infancia (pp. 99-120). UNESCO.

Magendzo, A. (2012). Algunos principios pedagógicos orientadores de la educación en derechos humanos. Revista Decisión, 32, 9-13. 
Magendzo, A. y Bermúdez, A. (2017). Pensando la educación en derechos humanos desde una mirada ética y controversial. Revista Latinoamericana de Derechos Humanos, 8(2), 17-33.

https://doi.org/10.15359/rldh.28-2.1

Martínez Sainz, G. (2018). Human rights education and training programs in Mexico: A cross-case analysis of practitioners' professional knowledge and practices. Mexican Law Review, 11(1), 103-130. https://doi.org/10.22201/iij.24485306e.2018.1.12513

Mercier, C. (2017, diciembre). ¿Cómo narrar la experiencia chilena? Estrategias literarias en el desierto de Carlos Franz, Nocturno de Chile de Roberto Bolaño y formas de volver a casa de Alejandro Zambra. Comunicación presentada en la VII Conferencia Internacional de Educación en Derechos Humanos (IHREC). Santiago de Chile. https://www.uchile.cl/publicaciones/139918/vii-conferencia-internacional-de-educacion-en-derechos-humanos-ihrec

Morales P. y Sandoval, V. (2017). Experiencia de formación de educadoras en el parque por la paz Villa Grimaldi. Cuadernos de Trabajos Educativos, 9(4), 94-107.

Naciones Unidas. (2019). Informe sobre la misión a Chile del 30 de octubre al 22 de noviembre del 2019. https://www. ohchr.org/Documents/Countries/CL/Report_Chile_2019_SP.pdf

Naciones Unidas. (2013). Promoción y protección de todos los derechos humanos, civiles, políticos, económicos, sociales y culturales, incluido el derecho al desarrollo. Informe del relator especial sobre la situación de los derechos humanos y las libertades fundamentales de los indígenas, Sr. Rodolfo Stavenhagen. https://www.acnur.org/fileadmin/Documentos/BDL/2008/6017.pdf

Naciones Unidas. (2012). Programa mundial para la educación en derechos humanos. Plan de acción. https://www.ohchr.org/Documents/Publications/WPHRE_Phase_2_sp.pdf

Naciones Unidas. (1996). Informe del alto comisionado de las Naciones Unidas para los derechos humanos sobre la aplicación del plan de acción para el decenio de las Naciones Unidas para la educación en la esfera de los derechos humanos. https://documentsddsny.un.org/doc/UNDOC/GEN/N97/008/05/PDF/N9700805.pdf?OpenElement

Naciones Unidas. (1993). Declaración y programa de acción de Viena. 20 años trabajando por tus derechos. Conferencia mundial de derechos humanos. https:/www.ohchr.org/Documents/Events/OHCHR20/VDPA_booklet_Spanish.pdf

Nin, M. C. y Lorda, M. A. (2019). Políticas educativas que propician la educación en memoria y derechos humanos. Revista Universitaria de Geografía, 28(1), 135-153.

Osorio, J. (2014). Educación para la ciudadanía: Debates pedagógicos sobre el aprendizaje democrático y de los derechos humanos en Chile y América Latina. Temas de Educación, 20(2), 25-36.

Paz Maldonado, E. y Díaz Pérez, W. (2019). Educación para la paz: Una mirada desde la Universidad Nacional Autónoma de Honduras. Innovación Educativa, 19(79), 1665-2673.

Quintar, E. (2009). Enseñanza como puente a la vida. Instituto Politécnico Nacional.

Rodino, A. M. (2015). La educación con enfoque de derechos humanos como práctica constructora de inclusión social. Revista IIDH, 61, 201-224.

Ruz, J. (1992a). La actitud teórica de las ciencias de la educación: Análisis y propuestas. Revista de Estudios Sociales, 71, 33-57.

Ruz, J. (1992b). Lo instrumental y lo valórico en la educación chilena. Revista de Estudios Sociales, 74, 45-76.

Santibáñez, B. (2017). Educar en derechos humanos en países post-conflicto: El caso chileno. Cuadernos de Trabajos Educativos, 9(4), 35-55.

Siemon, J. (2017). Testimonios en la educación en derechos humanos y memoria. Cuadernos de Trabajos Educativos, 9(4), 77-93.

Silva, C. e Ibáñez, A. (2015). Guía de educación en derechos humanos. Organización de Estados Iberoamericanos.

Stake, R. E. (2007). Investigación con estudio de caso. Morata. 
Toro, J. (2017). Educación en derechos humanos en el tratamiento del periodo de dictadura militar en Chile en sexto año básico. Cuadernos de Trabajos Educativos, 9(4), 56-63.

Torres, M. F. (2019). Educación para la paz y formación profesional: Aproximación desde la investigación documental. Praxis \& Saber, 10(22), 143-167. https://doi.org/10.19053/22160159.v10.n22.2019.7424

Trimiño, C. y Amézquita, L. (2018). Reflexiones desde la universidad sobre educación en derechos humanos y para la paz. Revista Historia de la Educación Latinoamericana, 20(31), 101-124. https://doi.org/10.19053/01227238.8564

Universidad Católica Silva Henríquez. e Instituto Nacional de Derechos Humanos. (2018). Programa diplomado de educación en derechos humanos para profesionales de la educación. https://www.indh.cl/bb/wp-content/uploads/2018/06/Programa-Diplomado-en-Educaci\%C3\%B3n-en-Derechos-Humanos-UCSH-INDH-2018.pdf

Universidad Católica Silva Henríquez. (2019). Resolución no 2019/024 deroga resolución de rectoría n 2016/058 y aprueba nueva Política de Investigación de la Universidad Católica Silva Henríquez.

http://ww3.ucsh.cl/wp-content/uploads/documentacion/politicas/Politica_institucional_de_investigacion.pdf

Valderrama Núñez, C. M. (2020). Alcances y desafíos de la educación en derechos humanos en la formación de terapeutas ocupacionales a partir de la percepción de los estudiantes. Cadernos Brasileiros de Terapia Ocupacional, 10,1-24. https://doi.org/10.4322/2526-8910.ctoao1828

Veneros Ruiz-Tagle, D. y Toledo Jofré, M. I. (2009). El uso pedagógico de lugares de memoria: Visita de estudiantes de educación media al parque por la paz Villa Grimaldi (Santiago, Chile). Estudios Pedagógicos, 35(1), 199-220. https://doi.org/10.4067/S0718-07052009000100012

Vega-Gutiérrez, A. M. y Navaridas-Nalda, F. (2018). Formación en derechos humanos en la educación superior. Global Social Work, 8, 35-56. https://doi.org/10.30827/tsg-gsw.v8i0.7432

Villegas, M., Newfarmer, L. y Francesconi, L. (2017). Libro de actas del VII IHREC 2016. VII Conferencia internacional de educación en derechos humanos. Los desafíos de la sociedad civil. Universidad de Chile.

Zemelman, H. (1992). Los horizontes de la razón. Antrophos.

Zemelman, H. (2010). Sujeto y subjetividad: La problemática de las alternativas como construcción posible. POLIS, Revista Latinoamericana, 9(27), 17-34.

https://doi.org/10.4067/S0718-65682010000300016

Zemelman, H. (2011). Configuraciones críticas. Pensar epistémico sobre la realidad. Siglo XXI.

\section{Breve CV de los/as autores/as}

\section{Carlos Bustos}

Profesor Titular de Historia e Investigador del Centro de Investigación para la transformación Socioeducativa de la Universidad Católica Silva Henríquez (UCSH). Doctor en Ciencias de la Educación Mención Interculturalidad. Coordinador de Ciencias Sociales para la carrera de Pedagogía en Educación Básica. Coordinador Académico del Diplomado de Educación en Derechos Humanos de la UCSH y el Instituto Nacional de Derechos Humanos. Miembro del Centro de investigación en Educación para la Justicia Social (CIEJUS) y de Instituto de Pensamiento y Cultura Latinoamericano (IPECAL). Email: cbustosr@ucsh.cl

ORCID ID: https://orcid.org/0000-0001-7090-7140

\section{Silvia Castillo}

Profesora de castellano por la Universidad Metropolitana de Ciencias de la Educación, Magíster en Lingüística por la Universidad de Chile y Doctora en Ciencias de la Educación, mención Interculturalidad 
por la Universidad de Santiago de Chile. Ha participado en iniciativas de enseñanza y revitalización del mapudungun y ha desarrollado investigaciones vinculadas a los procesos de enseñanza-aprendizaje de la lengua mapuche en contexto educativo. Coautora de We Pichikeche (Nuevos niños). Estrategias didácticas y pedagógicas para jardines interculturales en contexto urbano (2013), y de otros escritos publicados en revistas nacionales e internacionales. Email: profsilviacastillo@gmail.com

ORCID ID: https://orcid.org/0000-0003-2820-9511

\section{Simona Mayo}

Licenciada en Lengua y Literatura Hispánica con mención en Literatura de la Universidad de Chile. Magíster en Educación, Pedagogías Críticas y Problemáticas Socioeducativas de la Universidad de Buenos Aires. Estudiante del Doctorado en Lingüística de la Universidad de Buenos Aires. Se ha desempeñado en la enseñanza, revitalización y difusión del mapudungun en diversos espacios en Chile y Argentina y en la producción de material de enseñanza contextualizado a la realidad urbana y actual de la lengua mapuche. Email: simonna.mayo@gmail.com

ORCID ID: https://orcid.org/0000-0002-1775-6141

\section{Jorge Soto}

Sociólogo, Máster en Investigación en Sociología Aplicada por la Universidad Autónoma de Barcelona. Actualmente es coordinador de titulación y docente en el área de metodología del Magister en Desarro1lo Cognitivo del Centro de Desarrollo Cognitivo de la Universidad Diego Portales. Posee experiencia en investigación, consultoría y asesoramiento en temáticas de educación intercultural e inclusión educativa entre otros para Mineduc, Unicef y Unesco. Email: jorge.sotoc@mail.udp.cl

ORCID ID: https://orcid.org/0000-0001-5107-5668 